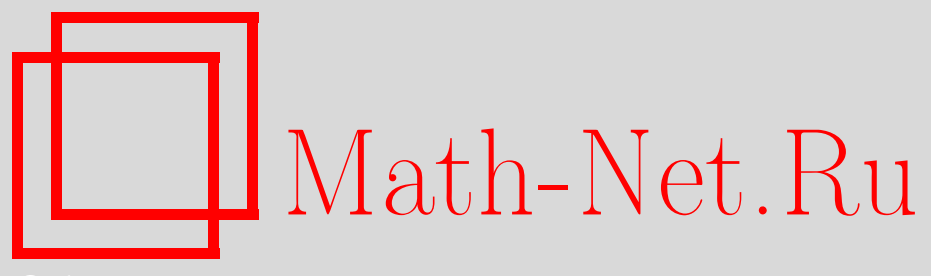

А. В. Пенской, Система Вольтерра и топология изоспектрального многообразия якобиевых матриц с нулевой диагональю, УМH, 2007, том 62, выпуск 3, 213-214

DOI: https://doi.org/10.4213/rm6808

Использование Общероссийского математического портала Math-Net.Ru подразумевает, что вы прочитали и согласны с пользовательским соглашением http://www . mathnet.ru/rus/agreement

Параметры загрузки:

IP : 54.147 .182 .235

26 апреля 2023 г., 10:17:41

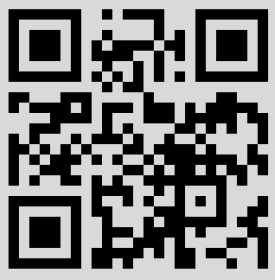




\section{Система Вольтерра и топология изоспектрального многообразия якобиевых матриц с нулевой диагональю}

\section{А. В. Пенской}

Рассмотрим симплектическое многообразие $\left(X^{2 n}, \omega\right)$ и интегрируемую систему с гамильтонианом $H$ и инволютивными интегралами $F_{1}=H, F_{2}, \ldots, F_{n}$. Пусть $X_{F} \subset$ $X$ - подмногообразие, заданное уравнениями $F_{1}=c_{1}, \ldots, F_{n}=c_{n}$. Это подмногообразие называется поверхностью уровня интегралов. Хорошо известная теорема Лиувилля-Арнольда [1] утверждает, что если $X_{F}$ компактно и связно, то это тор. Это делает изучение топологии $X_{F}$ тривиальным. Тем не менее, оказывается, что в некоторых важных примерах интегрируемых систем такое подмногообразие компактно, но его топология весьма сложна. Это происходит по причине того, что в этих примерах $X$ содержит точки, в которых или $H$ сингулярен, или $\omega$ сингулярна или вырождена, и теорема Лиувилля-Арнольда не применима. Тем не менее, в некоторых из этих ситуаций можно определить соответствующий поток на всем подмногообразии $X_{F}$ и успешно использовать его для исследования топологии $X_{F}$.

Первый пример подобного рода был описан К. Томеи в его работе [2], в которой исследована топология изоспектрального многообразия якобиевых матриц (т.е. вещественных трехдиагональных симметрических матриц). Это поверхность уровня интегралов (незамкнутой) цепочки Тоды. Используя поток Тоды, Томеи вычислил эйлерову характеристику этого многообразия. Двумя годами позже Д. Фрид [3] обнаружил, что стабильная и нестабильная стратификации, определенные потоком Тоды, являются клеточными комплексами. Используя этот факт, он нашел кольцо когомологий этого изоспектрального многообразия.

Нашей целью является изучение топологии изоспектрального многообразия вещественных якобиевых матриц с нулевой диагональю. Рассмотрим многообразие $M_{k}$ всех $(k \times k)$-матриц $L$ с фиксированным спектром вида $L_{i j}=c_{i} \delta_{i+1, j}+c_{i-1} \delta_{i-1, j}$.

ПредЛОЖение 1. а) Собственные числа такой матрицы имеют вид 0 (если $k$ нечетно), $\pm \lambda_{1}, \pm \lambda_{2}, \ldots$. b) Если все собственные числа различны, то $M_{k}$ - компактное гладкое многообразие и его топология не зависит от собственных чисел.

ДокАзАтельство. а) ясно. Доказательство b) аналогично доказательству Томеи подобного утверждения для изоспектрального многообразия якобиевых матриц [2].

Многообразие $M_{k}$ является поверхностью уровня интегралов (незамкнутой) системы Вольтерра $\dot{c}_{i}=\frac{1}{2} c_{i}\left(c_{i+1}^{2}-c_{i-1}^{2}\right)$, где $c_{0}=c_{k}=0$. Обычно эта система записывается в переменных $u_{i}=c_{i}^{2}$. Хорошо известно, что система Вольтерра может быть записана в лаксовой форме $\dot{L}=[L, A(L)]$. Из этой лаксовой формы следует, что поток Вольтерра сохраняет спектр $L$, т.е. поток Вольтерра является потоком на $M_{k}$. Хорошо известно [4], что система Вольтерра является интегрируемой. В четном $(k=2 l)$ случае $M_{k}$ имеет $2^{l}$ изоморфных компонент связности. Более того, каждая компонента изоморфна изоспектральному многообразию якобиевых матриц, изучавшемуся Томеи и Фридом, см., например, [5], [6]. Нечетный случай $(k=2 l+1)$ совершенно другой, $M_{k}$ связно. В настоящее время не ясно, как вычислять группы гомологий. Стабильная и нестабильная стратификации не являются клеточными комплексами в этом случае, и невозможно применить прямой подход, подобный подходу Фрида [3]. Мы должны также сказать, что в 1992 г. Э. Блох, Р. Брокетт и Т. Ратью [7] использовали идею двойного скобочного представления, чтобы показать, что цепочка Тоды является градиентным потоком. В 1998 г. [8] было показано, что поток Вольтерра также является градиентным потоком. Это приводит нас к естественной идее найти группы гомологий, используя комплекс Морса. K сожалению, мы не можем использовать комплекс Морса для нахождения групп гомологий, так как стабильная и нестабильная стратификации не трансверсальны друг другу. В настоящей работе мы вычисляем эйлерову характеристику $M_{k}$, используя поток Вольтерра. Подход близок к подходу Томеи [2]. 
Пусть $K=\frac{1}{4} \operatorname{diag}(1,2,3, \ldots)$ и $f(L)=\operatorname{tr} K L^{2}$. Доказано [8], что (отрицательный) градиентный поток $f(L)$ относительно некоторой метрики совпадает с потоком Вольтерра. Выберем $\lambda_{i}$ (см. предложение 1) таким образом, что все $\lambda_{i}>0$ и $\lambda_{i}>\lambda_{i+1}$.

Предложение 2. а) Критические точки $f(L)$ на $M_{2 l+1}$ находятся во взаимно однозначном соответствии с тройками $[j, s, \pi]$, состоящими из числа $j, 0 \leqslant j \leqslant l$, набора из $l$ чисел $s=\left(s_{1}, \ldots, s_{l}\right)$, где каждое $s_{i}$ равно 0 или 1, и перестановки $\pi \in S_{l}$. b) Индекс критической точки, соответствующей $[j, s, \pi]$, равен количеству $i, 0 \leqslant$ $i \leqslant l-1$, maких, что $i \neq j, j-1$ u $\pi(i)<\pi(i+1)$, плюс 1, если $j \neq l$.

ДокАЗАтЕЛЬСтво. Критические точки суть точки равновесия потока Вольтерра. Используя этот факт, а также явные полиномиальные уравнения $M_{k}$, можно доказать, что критические точки - в точности такие точки, что в точности $l$ из $2 l$ величин $c_{1}, \ldots, c_{2 l}$ равны нулю, с дополнительным свойством, что если $c_{i} \neq 0$, то $c_{i-1}=c_{i+1}=0$. Смотря на спектр соответствующей матрицы, получаем описание, данное в формулировке предложения. Это доказывает а). Формулу b) для индекса можно получить, изучая гессиан $f(L)$.

ПредЛоЖение 3. а) Эйлерова характеристика $M_{2 l+1}$ равна $\chi\left(M_{2 l+1}\right)=2^{2 l+2} \times$ $\left(2^{l+2}-1\right) \frac{B_{l+2}}{l+2}$, где $B_{l+2}-$ число Бернулли. b) Положим $\chi\left(M_{1}\right)=0$. Тогда экспоненциальная производящая функция равна $-\operatorname{th}^{2}(2 z)$, m.е. $-\operatorname{th}^{2}(2 z)=\sum_{l \geqslant 0} \chi\left(M_{2 l+1}\right) z^{l} / l !$.

ДоказАтельство. Напомним, что интервал $[i, i+1]$ такой, что $\pi(i)<\pi(i+1)$, называется подъемом. Число перестановок из $n$ элементов с $k$ подъемами называется числом Эйлера $\left\langle\begin{array}{l}n \\ k\end{array}\right\rangle$, см. [9]. Обозначим число подъемов в $\pi$ через $p(\pi)$. Пусть $\psi(n)=$ $\sum_{m=0}^{n}(-1)^{m}\left\langle\begin{array}{l}n \\ k\end{array}\right\rangle$, тогда из формулы (7.56) в [9] следует, что $1+$ th $z=\sum_{n \geqslant 0} \psi(n) z^{n} / n$ !. Из предложения 2 следует, что $\chi\left(M_{2 l+1}\right)$ равна

$$
\begin{gathered}
2^{l} \sum_{j=1}^{l-1}\left(\begin{array}{l}
l \\
j
\end{array}\right) \sum_{\pi_{1} \in S_{j}, \pi_{2} \in S_{l-j}}(-1)^{p\left(\pi_{1}\right)+p\left(\pi_{2}\right)+1}=-2^{l} \sum_{j=1}^{l-1}\left(\begin{array}{l}
l \\
j
\end{array}\right)\left(\sum_{\pi_{1} \in S_{j}}(-1)^{p\left(\pi_{1}\right)}\right) \\
\times\left(\sum_{\pi_{2} \in S_{l-j}}(-1)^{p\left(\pi_{2}\right)}\right)=-2^{l} \sum_{j=1}^{l-1}\left(\begin{array}{l}
l \\
j
\end{array}\right) \psi(j) \psi(l-j) .
\end{gathered}
$$

Из этого следует b). Из разложения th $z$ и формулы $\operatorname{th}^{\prime} z=1-\operatorname{th}^{2} z$, получаем а).

Автор благодарен А.П. Веселову за постановку задачи, а также О. Корнеа и А. Медведовской за плодотворные обсуждения.

\section{Список литературы}

[1] В. И. Арнольд, Математические методы классической механики, Наука, М., 1989. [2] C. Tomei, Duke Math. J., 51:4 (1984), 981-996. [3] D. Fried, Proc. Amer. Math. Soc., 98:2 (1986), 363-368. [4] С. В. Манаков, ЖЭЭТФ, 67:2 (1974), 543-555. [5] Р. А. Damianou, Phys. Lett. A, 155:2-3 (1991), 126-132. [6] В. Л. Верещагин, Матем. заметки, 48:2 (1990), 145148. [7] A. M. Bloch, R.W. Brockett, T. S. Ratiu, Comm. Math. Phys., 147:1 (1992), 57-74. [8] A. V. Penskoi, Regul. Chaotic Dyn., 3:1 (1998), 76-77. [9] Р. Грэхем, Д. Кнут, О. Паташник, Конкретнал математика: Основание информатики, Мир, М., 1998.

\section{А. В. Пенской (А. V. Penskoi)}

Независимый московский университет МГТУ им. Н. Э. Баумана

E-mail: penskoi@mccme.ru
Представлено А. В. Михалёвым Принято редколлегией 27.03.2007 